\title{
BMJ
}

\section{Effect of general practitioner education on adherence to antihypertensive drugs: cluster randomised controlled trial}

\author{
Nudrat Noor Qureshi, research fellow, Juanita Hatcher, associate professor, ${ }^{1}$ Nish Chaturvedi, professor, ${ }^{2}$ \\ Tazeen $\mathrm{H}$ Jafar, associate professor, ${ }^{1}$ for the Hypertension Research Group
}

${ }^{1}$ Clinical Epidemiology Unit, Department of Community Health Sciences, Aga Khan University, P O Box 3500, Stadium Road, Karachi, 74800, Pakistan

${ }^{2}$ International Centre for Circulatory Heath,

National Heart and Lung Institute, Imperial College, London

Correspondence to: $\mathrm{T} \mathrm{H}$ Jafar

tazeen.jafar@aku.edu

doi:10.1136/bmj.39360.617986.AE

\section{ABSTRACT}

Objective To determine the impact of a simple educational package for general practitioners on adherence to antihypertensive drugs.

Design Cluster randomised controlled trial.

Setting Six randomly selected communities in Karachi, Pakistan.

Participants 200 patients with hypertension taking antihypertensive drugs; 78 general practitioners. Intervention Care by general practitioners specially trained in management of hypertension compared with usual care.

Main outcome measure Correct dosing, defined as percentage of prescribed doses taken, measured with electronic medication event monitoring system (MEMS) bottle.

Results 200 patients were enrolled, and 178 (89\%)

successfully completed six weeks of follow-up. Adherence was significantly greater in the special care group than in the usual care group (unadjusted mean percentage days with correct dose $48.1 \%, 95 \%$ confidence interval $35.8 \%$ to $60.4 \%$, versus $32.4 \%$, $22.6 \%$ to $42.3 \% ; \mathrm{P}=0.048$ ). Adherence was also higher among patients who had higher levels of education ( $P<0.001)$, were encouraged by family members $(P<0.001)$, believed in the effect of drugs $(P<0.001)$, and had the purpose of the drugs explained to them $(P<0.001)$. Conclusions Special training of general practitioners in management of hypertension, emphasising good communication between doctors and patients, is more effective than usual care provided in the communities in Karachi. Such simple interventions should be adopted by other developing countries that are now facing an increasing burden of hypertension.

Trial registration Clinical trials NCT00330408.

\section{INTRODUCTION}

Hypertension is one of the most common risk factors for cardiovascular disease and has one of the highest attributable risks for death worldwide. ${ }^{12}$ Despite well established benefits of lowering blood pressure, and the existence of several national and international guidelines on detection and management of hypertension, control remains poor. ${ }^{3}$ Half of patients with hypertension in the United States reported receiving drugs for lowering blood pressure. However, only
$30 \%$ had their blood pressure controlled to the conventionally recommended target of less than 140/90 mm $\mathrm{Hg}$. ${ }^{4}$ The situation is much worse in developing countries, where the prevalence of hypertension is high and blood pressure control rates are extremely low-for example, about $6 \%$ in China and less than $3 \%$ in Pakistan. ${ }^{56}$

Suboptimal practice patterns by doctors, leading to inadequate adherence to antihypertensive drugs by patients, have been implicated as important contributors to poor blood pressure control. ${ }^{7-9}$ Our previous report on a national survey of general practitioners in Pakistan identified serious gaps in knowledge and practice regarding management of hypertension. ${ }^{10}$ Similar findings have been reported from developed countries. ${ }^{11-13}$

We did this study in Karachi, Pakistan, to assess the impact of special educational training of general practitioners in the management of hypertension on adherence to prescribed antihypertensive drugs and to determine the practice patterns of doctors that contribute to adherence to antihypertensive drugs. We hypothesised that adherence to antihypertensive drugs by patients seeking care from general practitioners specially trained in the management of hypertension would be higher than in those seeking care from general practitioners providing usual care.

\section{METHODS}

This study was a substudy of a cluster randomised controlled trial with a factorial design to determine the impact of family based home health education and specially trained general practitioners on blood pressure. However, the substudy was limited to the clusters not randomised to home health education, as this intervention was expected to have only a modest effect on adherence independent of training of general practitioners. The sampling frame has been described previously. ${ }^{14}$ In brief, using a multistage random sampling technique, we selected 12 out of 5000 geographical census based clusters in Karachi (250 households in each cluster). If a selected cluster was less than $10 \mathrm{~km}$ from a previously selected cluster, we replaced it with a new cluster to minimise the risk of contamination by the intervention. From these 12 clusters, we used computer generated codes to randomly assign 
three each to special care by a general practitioner, home health education, both home health education and special care, and usual care by a general practitioner. We chose the six clusters without home health education for this study. We needed a sample size of 109 participants in each group to detect a difference in adherence of $20 \%(\alpha=0.05, \beta=0.20)$, using a design effect of 1.5 to account for clustering by general practitioner, an estimated intraclass correlation of 0.015 , and a $75 \%$ response rate. ${ }^{15}$

\section{Intervention}

We invited all general practitioners located in clusters randomised to special care to attend a one day intensive training session on hypertension. This focused on standard treatment algorithms for the management of hypertension based on the seventh report of the Joint National Committee (JNC VII) and the report of the Fourth Working Party of the British Hypertension Society modified for the Indo-Asian population. ${ }^{1617}$

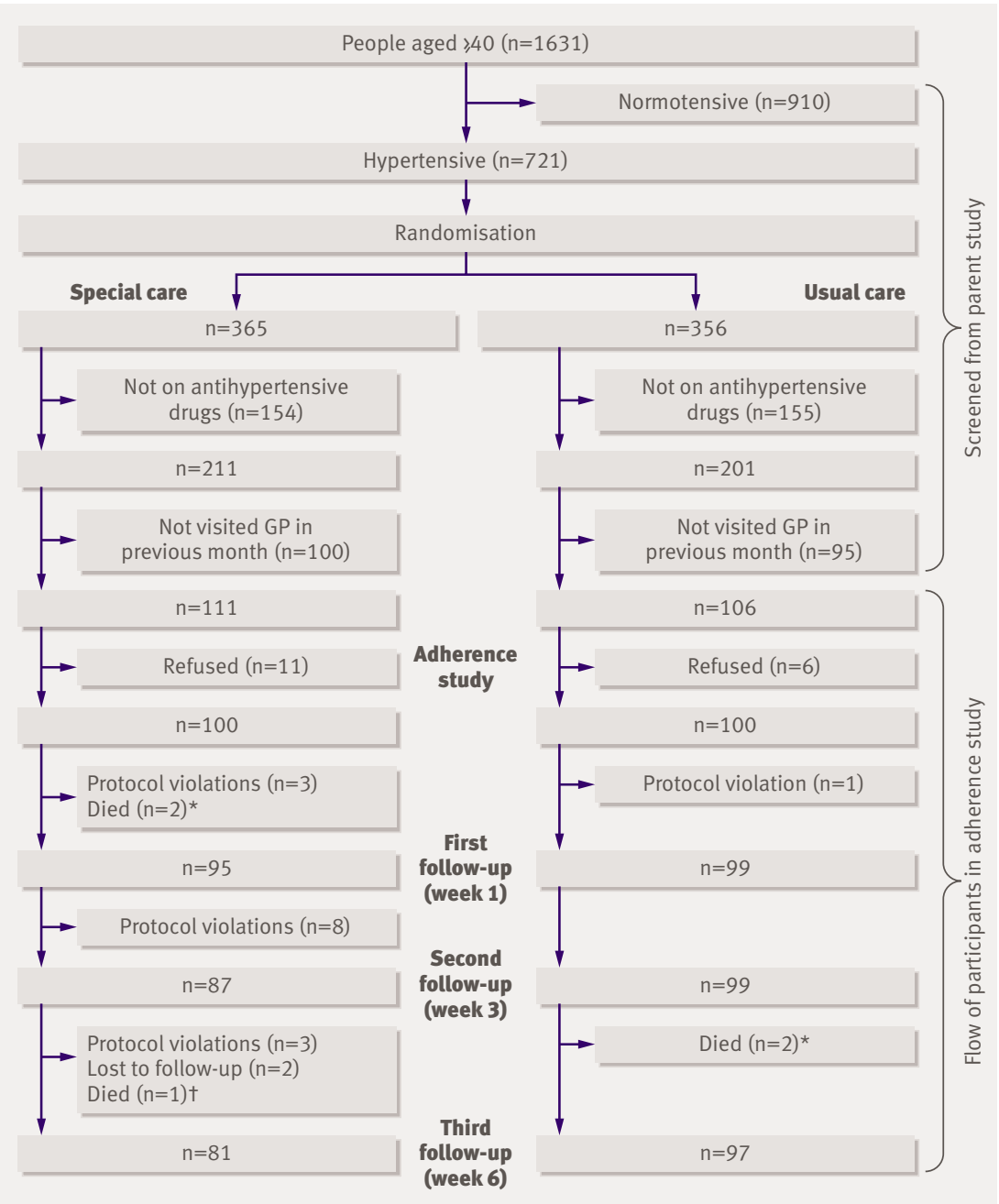

Study profile. Special care=care given by general practitioners (GPs) intensively trained in management of hypertension; usual care=standard care given by GPs in community. *Death from heart attack. †Death from stroke
Components of the course included non-pharmacological (diet, exercise, weight loss, smoking cessation) and pharmacological interventions; prescribing low cost and appropriate generic drugs; preferential use of single dose drug regimens; scheduled follow-up visits; stepped care approach for titration of drugs to achieve target blood pressure levels; and satisfactory consultation sessions for patients, with explanations of treatment and use of appropriate communication strategies. The recommended target blood pressure was less than $140 / 90 \mathrm{~mm} \mathrm{Hg}$ for all patients. Although this diverges from the recent guidelines for special subgroups (people with diabetes, those with end organ damage), we reasoned that we needed to keep the intervention, guidelines, and targets simple for both patients and practitioners in a setting in which blood pressure control rates are less than 3\%. Training sessions for general practitioners used case scenarios and were interactive. We provided blood pressure treatment manuals and easy to read mountable treatment charts. All general practitioners took pre-training and post-training examinations. A certificate of training was offered at the end of training.

\section{Inclusion and exclusion criteria}

Eligible participants were people residing in the selected clusters, were aged 40 years and above, had been identified as having hypertension in the parent project, had visited their general practitioner within the previous month, and had been prescribed antihypertensive drugs (that is, those in whom adherence could be assessed with reasonable accuracy). We excluded pregnant women, people unable to give consent, and bed bound patients.

\section{Screening visit (parent study)}

In the parent study, trained community health workers paid home visits to all homes in the selected cluster. They used a structured questionnaire to collect demographic, lifestyle, and medical information on all people who gave informed consent. They measured blood pressure twice, 20 minutes apart, with a calibrated automated device (Omron HEM-737 BP Monitor) in the sitting position after five minutes of rest. People with high blood pressure, and not taking antihypertensive drugs, were visited again for re-measurement of blood pressure. All measurements were made to a standard protocol that conforms to the international standards for definitions and measurements. People with hypertension (mean systolic pressure $\geq 140 \mathrm{~mm} \mathrm{Hg}$ or mean diastolic pressure $\geq 90 \mathrm{~mm} \mathrm{Hg}$ on both visits, or taking antihypertensive drugs) were advised to consult a local general practitioner. Participants in the special care group were given a list of specially trained general practitioners within their cluster to choose from. Participants were blinded to intervention status (training of general practitioner). 
Recruitment visit (adherence study)

Recruitment to the adherence study started six months after the screening visit. We sought informed consent for this study. We used a standardised questionnaire to collect information from the patients on their demographics and attitudes and on doctors' practice patterns. This questionnaire was informed by other international surveys and was rigorously pretested on 20 participants in a similar population to the study population. ${ }^{1819}$ Trained community health workers provided medication event monitoring system (MEMS) bottles (Aardex, Switzerland), an electronic monitor that records the exact time and date of each cap opening sequence, with detailed instructions on the use and storage of the bottles. ${ }^{2021}$ Patients taking one type of antihypertensive drug were given one bottle and those taking two were provided with two separate electronic monitors.

\section{Follow-up visits}

Community health workers followed up all participants on three occasions for a total of six weeks, at the end of weeks one, three, and six after recruitment. At each follow-up visit, they measured adherence to drugs by downloading data from the MEMS on to a computer with a communicator device and using PowerView software to summarise the data. The MEMS were reissued to participants at each followup visit, and participants were asked about any unintentional openings of the cap. This information was recorded on the data form to minimise the reporting of false events. Protocol violators were those who failed to use the MEMS as advised, either owing to difficulty in opening the caps or by taking prescribed drugs from the leaf instead of the assigned bottles. Outcome assessors were blinded to the randomisation status of participants.

At the final visit, community health workers trained in standardised measurements of blood pressure measured blood pressure in a subset of 112 randomly selected patients ( 55 from the special care group and 57 from the usual care group), according to the same method as in the screening visit. These community health workers were also masked to the randomisation status.

\section{Statistical analysis}

The primary outcome was "correct dosing of drugs," defined as percentage of prescribed doses taken during the monitored interval. This was updated on all three follow-up visits over six weeks, and we used all available values for the analyses.

We used SAS version 9.1 for statistical analyses, accounting for clustering at the level of the general practitioner within each cluster. ${ }^{22}$ We compared the distributions of patient related variables between those who were randomised to special care versus usual care by using analysis of variance for continuous variables and the $\chi^{2}$ test for discrete variables. We used nested mixed model analyses of variance to test for significance of the effect of special care versus usual care on the primary outcome. ${ }^{23} \mathrm{We}$ followed the intention to treat principle. We analysed data on all patients enrolled at the start of the study in the two randomised groups for the time they were followed in the study $(\mathrm{n}=200)$.

We tested patient related and doctor related factors and retained them in the models if they were significantly associated with adherence. Candidate patient related factors were sex, age, educational status, occupation, marital status, socioeconomic status, family type (extended versus nuclear), and belief in the value of antihypertensive drugs. Doctor related factors were consultation time, the cost and frequency of drugs, explaining the name and the purpose of the drugs, and the doctor's attitude (satisfactory or not). We considered a $\mathrm{P}$ value of $<0.05$ to be statistically significant. We report means and 95\% confidence intervals for each of the significant factors, adjusted to the mean values of the other variables in the model. We developed two models - one including only the patient related factors and the second including both patient and doctor related factors to explore the components of the training that were significant.

We did sensitivity analyses on participants who successfully completed the six week follow-up as per protocol $(n=178)$, restricted to those without missing information on adherence, and on the entire dataset $(n=200)$ after substituting missing values with the unadjusted and adjusted mean adherence values obtained in the usual care and special care groups. We determined the lowest level of adherence for missing values for which the adjusted difference between the two groups was still significant.

We also did a supplementary analysis on a subset of 112 patients to assess the effect of improved adherence (defined as those who took at least $50 \%$ of the prescribed drugs, based on the top third of patients for adherence) on blood pressure. The blood pressure readings in these patients were taken as part of the parent study within a month of the last visit for measurement of adherence.

\section{RESULTS}

Of the 1685 households in the six clusters included in this study, 1560 households agreed to participate in the parent study (response rate $=92.5 \%$ ). From these households, 1631 people were aged 40 and above, of whom $721(44.2 \%)$ had hypertension (figure). During the recruitment visit for the adherence study, $217(30 \%)$ of these people with hypertension reported having visited their general practitioner during the previous month and being prescribed antihypertensive drugs. These people were eligible for enrolment; $200(92.1 \%)$ of them consented to participate in the study. We analysed data on these participants on the intention to treat principle.

During follow-up visits, five deaths occurred (three in the special care group-one from stroke, two from heart attack; two in the usual care group-both from heart attack; $\mathrm{P}=0.50), 15$ participants were withdrawn from the study owing to protocol violations (14 in 
Table 1 | Distribution of patient related and doctor related characteristics in special care and usual care groups. Values are numbers (percentages) unless stated otherwise

\section{Variables}

Patient related characteristics

Mean $(95 \% \mathrm{Cl})$ age (years)

Sex:

\section{Male}

Female

Monthly household income (US\$):

\begin{tabular}{l}
$25-<75$ \\
\hline $75-<116$ \\
\hline $116-200$ \\
\hline$>200$ \\
Education status::
\end{tabular}

\section{Illiterate}

Primary school

Secondary school

Graduate and above

Currently employed:

\begin{tabular}{l} 
Yes \\
No \\
\hline Marital status: \\
\hline Married \\
\hline Widowed or single \\
\hline Family type§: \\
\hline Nuclear \\
\hline Extended \\
\hline Encouraged by family to take drugs: \\
\hline Yes \\
\hline No \\
\hline Belief in taking antihypertensive drugs:
\end{tabular}

Belief in taking antihypertensive drugs:

\begin{tabular}{ll}
\hline Yes \\
No \\
\hline Diabetes:
\end{tabular}

Diabetes:

\begin{tabular}{|c|c|c|c|c|}
\hline Yes & $53(26.5)$ & 26 & 27 & \multirow{2}{*}{0.822} \\
\hline No & $147(73.5)$ & 74 & 73 & \\
\hline \multicolumn{5}{|l|}{ Accompanied to clinic by family member: } \\
\hline Yes & $128(64.0)$ & 63 & 65 & \multirow{2}{*}{0.840} \\
\hline No & $72(36.0)$ & 37 & 35 & \\
\hline \multicolumn{5}{|l|}{ Antihypertensive drugs perceived as costlyף: } \\
\hline Yes & $152(76.0)$ & 71 & 81 & \multirow{2}{*}{0.011} \\
\hline No & $48(24.0)$ & 29 & 19 & \\
\hline \multicolumn{5}{|l|}{ Doctor related characteristics } \\
\hline Mean $(95 \% \mathrm{Cl})$ monthly cost of antihypertensive drugs (US\$) & $178(89.0)$ & $4.67(4.2-5.1)$ & $4.87(4.6-5.0)$ & 0.981 \\
\hline \multicolumn{5}{|l|}{ Frequency of prescribed drug: } \\
\hline Once a day & $54(27.0)$ & 28 & 26 & \multirow{2}{*}{0.742} \\
\hline Twice a day & $146(73.0)$ & 72 & 74 & \\
\hline \multicolumn{5}{|l|}{ Satisfactory attitude of doctor: } \\
\hline Yes & $179(89.5)$ & 91 & 88 & \multirow{2}{*}{0.369} \\
\hline No & $21(10.5)$ & 9 & 12 & \\
\hline \multicolumn{5}{|l|}{ Patient told of drug name: } \\
\hline Yes & $171(85.5)$ & 86 & 85 & \multirow{2}{*}{0.833} \\
\hline No & $29(14.5)$ & 14 & 15 & \\
\hline \multicolumn{5}{|l|}{ Explained purpose of drug(s) to patient: } \\
\hline Yes & $54(27.0)$ & 37 & 17 & \multirow{2}{*}{0.013} \\
\hline No & $146(73.0)$ & 63 & 83 & \\
\hline \multicolumn{5}{|l|}{ Self reported consultation time (minutes): } \\
\hline$\ll 10$ & $84(42.0)$ & 35 & 49 & \multirow{2}{*}{0.038} \\
\hline$\geq 10$ & $116(58.0)$ & 65 & 51 & \\
\hline
\end{tabular}

*Care given by general practitioners intensively trained in management of hypertension.

tUsual care given by general practitioners in the community.

†Primary $=1-5$ years of schooling; secondary $=6-12$ years of schooling; graduate or above $=12$ years of schooling or above.

§Nuclear family defined as household consisting of two parents and their children; extended family defined as household in which multiple generations of a family were living together.

TSelf reported to assess whether patient finds drugs prescribed by doctor costly.

\begin{tabular}{clll}
$75(37.5)$ & 36 & 39 & \multirow{2}{*}{0.680} \\
\cline { 1 - 3 } $125(62.5)$ & 64 & 61 & \\
\hline
\end{tabular}

\begin{tabular}{|c|c|c|c|}
\hline $43(21.5)$ & 25 & 18 & \multirow{3}{*}{0.312} \\
\hline $55(27.5)$ & 28 & 27 & \\
\hline $43(21.5)$ & 20 & 23 & \\
\hline
\end{tabular}

$50(25.0)$

$71(35.5)$

$60(30.0)$

$19(9.5)$

$\begin{array}{ll}24 & 26 \\ 34 & 37 \\ 31 & 29 \\ 11 & 8\end{array}$

78 (39.0)

$42 \quad 36$

$122(61.0)$

58

64

0.416

159 (79.5)

$41(20.5)$

86

$125(62.5)$

61

75 (37.5)

39

36

0.740

$156(78.0)$

79

77

$44(22.0)$

21

23

0.740

$167(83.5)$

$33(16.5)$

84

83

16

0.600

$53(26.5)$

$128(64.0)$

$152(76.0)$

$178(89.0)$

.742

$54(27.0)$

179 (89.5)

171 (85.5)

86

$54(27.0)$

$84(42.0)$

65 
special care group; 1 in usual care group; $\mathrm{P}=0.001)$, and two participants (both in special care group; $\mathrm{P}=0.49$ ) were lost to follow-up. Thus, 178 participants $(81$ in special care group; 97 in usual care group) successfully completed six weeks of follow-up.

A total of 36 out of 55 invited general practitioners in the special care group received the training programme. The average score in the pre-training examination was $32.3 \%$; this increased to $63.2 \%$ after training $(\mathrm{P}<0.001)$. The average time lag between training of doctors and referral of participants was six months; $98(98 \%)$ patients in the special care group sought care from the 36 trained general practitioner. The training status of the general practitioner attending to one patient in the special care group could not be verified. Patients in the usual care group were treated by 42 general practitioners. Thus, the patients were treated by 78 general practitioners: 36 in the special care group and 42 in the usual care group.

Patients' characteristics did not differ by randomisation group (table 1). Among the doctor related characteristics, the reported consultation time was more likely to be 10 minutes or more in the special care group than in the usual care group $(65 \% v 51 \% ; \mathrm{P}=0.04)$ (table 1). The general practitioner was more likely to explain the purpose of the drug to the patient in the special care group $(37 \% v 17 \% ; \mathrm{P}=0.01)$. Notably, drug costs did not differ between the groups.

The intraclass correlation coefficient for adherence to antihypertensive drugs for clustering at the general practitioner level was 0.06 (95\% confidence interval 0.00 to 0.16 ). Patients randomised to special care took a greater percentage of the prescribed drugs than those randomised to usual care $(48.1 \%, 95 \%$ confidence interval $35.8 \%$ to $60.4 \%$, versus $32.4 \%, 22.6 \%$ to $42.3 \%$; $\mathrm{P}=0.048$ ) (table 2). Key patient related factors that influenced correct dosing were the educational status of the patient, belief in taking drugs, and encouragement by the family to take the drug. The key doctor related practice was explaining the purpose of the $\operatorname{drug}(\mathrm{s})$ to the patient (table 2). In the multivariable model, these factors remained independent predictors of correct dosing. The $\mathrm{R}^{2}$ for the model was $73 \%$.

Sensitivity analysis showed consistent results for per protocol analysis in the dataset restricted to participants with all follow-up data $(n=178)$ (adjusted mean percentage days correct dose $49.8 \%, 38.4 \%$ to $61.1 \%$ for special care; $25.4 \%, 24.3 \%$ to $46.5 \%$ for usual care; $\mathrm{P}=0.02)$ and in the entire dataset $(\mathrm{n}=200)$ when we used imputed adherence levels ranging from $100 \%$ to $6 \%$ for those who withdrew from the study in both groups. In the subgroup analysis, the falls in systolic blood pressure $(8.3 \mathrm{~mm} \mathrm{Hg} ; \mathrm{P}=0.04)$ and diastolic blood pressure $(3.8 \mathrm{~mm} \mathrm{Hg} ; \mathrm{P}=0.1$ ) were greater among those with good adherence $(\geq 50 \%$ of drugs; $n=29)$ than those with poor adherence $(<50 \%$ of drugs; $\mathrm{n}=83)$.

\section{DISCUSSION}

To our knowledge, this is the first report of a community based randomised trial to assess the impact of special training of general practitioners in the management of hypertension on adherence to antihypertensive drugs. We found that relative adherence in people seeking care from specially trained general

Table 2 | Factors associated with adherence to antihypertensive drugs $(\mathrm{n}=\mathbf{2 0 0})$. Values are mean ( $95 \%$ confidence interval) percentage of days on which correct dose of drugs was taken

\begin{tabular}{|c|c|c|c|}
\hline \multirow[b]{2}{*}{ Variables } & \multicolumn{3}{|c|}{ Primary outcome: correct dosing* } \\
\hline & Univariate & Multivariable model $1 \dagger$ & Multivariable model $2 \ddagger$ \\
\hline Special care§: & $(P=0.048)$ & $(P=0.016)$ & $(P=0.030)$ \\
\hline Yes & 48.1 (35.8 to 60.4$)$ & 42.3 (30.6 to 53.9 ) & $42.8(30.8$ to 54.7$)$ \\
\hline No & $32.4(22.6$ to 42.3$)$ & $25.6(15.2$ to 36.0$)$ & 27.9 (19.7 to 47.9$)$ \\
\hline Education status: & $(P=0.001)$ & $(P<0.001)$ & $(P=0.001)$ \\
\hline Illiterate & $41.4(35.1$ to 47.6$)$ & 33.7 (27.9 to 39.6$)$ & $35.5(29.6$ to 41.4$)$ \\
\hline Primary school & $35.2(29.5$ to 40.9$)$ & 26.8 (21.5 to 32.1$)$ & 29.8 (24.0 to 35.6$)$ \\
\hline Secondary school & 41.5 (35.4 to 47.5$)$ & $27.6(21.2$ to 34.0$)$ & $29.2(22.8$ to 35.6$)$ \\
\hline Graduate and above & $56.7(47.0$ to 66.4$)$ & $47.6(39.1$ to 56.0$)$ & 46.9 (38.6 to 55.2$)$ \\
\hline Encouraged by family: & $(P<0.001)$ & $(P<0.001)$ & $(P<0.001)$ \\
\hline Yes & $44.5(40.9$ to 48.1$)$ & 41.1 (36.6 to 45.6$)$ & 42.1 (37.6 to 46.6$)$ \\
\hline No & 26.7 (20.2 to 33.1) & 26.7 (20.6 to 32.9) & $28.6(22.4$ to 34.8$)$ \\
\hline Belief in taking drugs to treat illness: & $(P<0.001)$ & $(P<0.001)$ & $(P<0.001)$ \\
\hline Yes & $44.5(41.0$ to 48.0$)$ & $43.6(39.4$ to 47.7$)$ & 44.9 (40.7 to 49.2$)$ \\
\hline No & 21.5 (13.3 to 29.8$)$ & 24.3 (17.0 to 31.5) & 25.8 (18.6 to 33.0$)$ \\
\hline Explaining purpose of drug(s) to patient: & $(P<0.001)$ & & $(P=0.023)$ \\
\hline Yes & 50.1 (43.9 to 56.3$)$ & - & 31.7 (27.2 to 36.2$)$ \\
\hline No & 36.7 (33.0 to 40.4$)$ & - & 34.7 (29.6 to 39.8$)$ \\
\hline \multicolumn{4}{|c|}{$\begin{array}{l}\text { *Defined as percentage of prescribed doses taken during monitored interval. } \\
\text { †Includes patient related factors. } \\
\text { †Includes patient related and training related factors. } \\
\text { §Care given by general practitioners intensively trained in management of hypertension. } \\
\text { P value for special care is based on } \mathrm{MS}_{(\text {(cluster) }} \text { as error term; } \mathrm{P} \text { values for all other variables are based on } \mathrm{MS}_{(\mathrm{error})} \text {. }\end{array}$} \\
\hline
\end{tabular}


practitioners was 50\% higher than in those consulting untrained general practitioners. Achieved blood pressure was lower in participants who adhered to their drugs than in those who did not. The main doctor related factor associated with increased adherence was explaining the purpose of the $\operatorname{drug}(\mathrm{s})$ to the patient. However, this factor alone did not account for the greater adherence (relative increase of $53 \% ; \mathrm{P}=0.03$ ) to drugs in the special care group, and other factors need to be sought.

Despite the increasing burden of mortality associated with chronic diseases, particularly in developing countries, reducing it remains a neglected millennium development goal. ${ }^{1-24}$ The national health survey of Pakistan (1990-4) highlighted the enormous burden of hypertension in Pakistan, with a prevalence of 33\% in people aged 45 and above. Moreover, more than $70 \%$ of people with hypertension were unaware of their condition, and less than $3 \%$ had adequately controlled blood pressure. ${ }^{625}$ The situation in neighbouring developing countries is quite similar. Trends show a rising prevalence of hypertension, reaching 24\% among people aged 35-59 years in China, but rates of awareness $(43 \%)$ and control $(6 \%)$ remain grossly inadequate..$^{5}$

The public health services in Pakistan, as in most other regional developing countries including India and China, are dysfunctional. ${ }^{2627}$ The vast majority of the population in these countries seek care from allopathic doctors; private general practitioners are the frontline care givers and are visited about five times annually by adults in Pakistan. ${ }^{625}$ A substantial portion of the cost of health care for patients in these countries is out of pocket, imposing a potential barrier to adherence to drugs. All drug classes are available in branded as well as generic forms. Previously, we reported marked deficiencies in the knowledge and approach of general practitioners in Pakistan relating to the management of high blood pressure and identified serious limitations in current practice, including the use of sedatives as first line antihypertensive agents by a quarter of general practitioner. ${ }^{10}$

Studies on doctor targeted interventions on hypertension control worldwide have tested several different strategies, with heterogeneous results. ${ }^{1819}$ Benefits of training doctors on implementation of guidelines and prescription practices have been documented. ${ }^{18}$ In the United States, the "hypertension specialist" programme of the American Society of Hypertension offers certification as "clinical specialist in hypertension" on verification of credentials and passing a qualifying examination based on a comprehensive specially designed curriculum. ${ }^{28}$ The hypertension clinics staffed by these specialists have had a positive impact on control of hypertension. ${ }^{29}$

\section{Implications of the study}

Our results support the value of such training programmes and show for the first time that even limited training of doctors leads to better adherence to antihypertensive drugs in a developing country setting. This is probably because of the potential room for improvement in terms of both training of general practitioners and its impact on patients' behaviour. On the basis of epidemiological evidence, this is likely to translate into improved cardiovascular morbidity and mortality from better control of blood pressure, provided the programme and its benefit are sustainable in the long term. Thus, inclusion of continuing medical education activities at a national level in programmes for prevention of chronic diseases in other developed and developing countries can be recommended.

We also found that significantly more general practitioners in the special care group than the usual care group were spending at least 10 minutes consulting with their patients. Although consultation time was not independently associated with adherence, this strategy was part of the training package, which as a whole was found to be beneficial. Despite limitations of self reported consultation times, the findings are consistent with qualitative assessments of doctors' perceptions of their practices on other health outcomes. ${ }^{30}$ The average consultation time with a general practitioner in the United Kingdom, where blood pressure control rates are less than $10 \%$, is about 9 . 4 minutes - twice as long in the United States, where control rates are $30 \% .{ }^{31-33}$ Healthcare planners need to keep these findings in perspective, given the tight budgets and clinic schedules of most public and private general practitioners worldwide.

In particular, our findings clearly underscore the importance of explaining the reasons for drugs in improving adherence, and this needs to be a key facet in clinical practice guidelines and training packages for general practitioners and other healthcare providers. Room for improvement also still exists in overall adherence, which was $48 \%$ among people seeking treatment from trained general practitioners. Other promising strategies, such as nurse led or pharmacist led care for hypertension control, could be potentially useful adjuncts for enhancing adherence. ${ }^{18}$ Their benefits in developing country settings remain to be explored.

\section{Limitations}

Our study had some potential limitations. Firstly, we did the study six months after the general practitioners were trained. This shows the persistence of a single education session in the medium term but does not test the long term beneficial effects. This needs to be tested formally; if attenuation does occur, this would support the need for follow-up training. We also need to assess whether long term impacts of changes in adherence and in blood pressure control translate to fewer cardiovascular events. Secondly, opening of the MEMS bottle does not necessarily ensure that the patient takes a pill, and the bottles are subject to potential manipulation. Constant reminders on how to use the bottles should have minimised this risk. Similarly, knowing that adherence is being assessed may inflate actual adherence. We would expect these 
potential effects to be balanced between randomised groups and not bias the conclusion.

Thirdly, inclusion of only participants who attended the general practice clinic within the previous month (30\% of hypertensive patients) introduces a selection bias against those who do not seek medical assistance. However, given that, on average, adults in Pakistan visit a general practitioner frequently, and that the characteristics of our study population are comparable to people with hypertension from the general population of Pakistan, we believe that our findings would be generalisable to the population at large. ${ }^{6}$ Fourthly, more patients withdrew from the special care group than the usual care group as a result of protocol violation. However, the results of sensitivity analyses indicated no difference in overall results in an intention to treat analysis that included the available data on all available visits as well as the per protocol analysis on those with complete follow-up and after imputation of missing data by adherence values that were much below the mean adherence observed in the usual care group. Thus, we believe our findings are robust.

Finally, information on doctors' practice factors was collected from the patients up until one month after their visit and may be subject to recall bias. Again, this should be equally balanced by randomisation. Moreover, we did not collect information on the demographics of the general practitioners, such as age, years since graduation, and length of practice. However, our purpose was to focus on modifiable doctor related factors potentially related to adherence. In addition, we did no external validation for either patient related or doctor related items on the questionnaire, and the study may not have had sufficient power to detect the association of some of these factors with adherence. However, the study was powered to find an important difference in the primary outcome.

\section{Strengths}

Strengths of our study include the use of a population based setting, random selection and randomisation of study groups, blinding of patients to treatment assignment, use of electronic MEMS bottles for measuring adherence, and measurement of adherence over a cumulative period of six weeks rather than at a single point in time. In addition, consistent with our previous experience, we saw high response rates, probably reflecting the eagerness on the part of this population in activities with perceived benefits to their health in an environment in which free health care is not available. ${ }^{614} \mathrm{We}$ think that these strengths increase the validity and generalisability of our findings.

Uptake of our training programme by general practitioners was high, which may result from several factors. Firstly, as general practitioners are private contractors, they are competing with each other for business. A certificate from a prestigious institution, such as the Aga Khan University, is viewed as an advantage; conversely, general practitioners who do not have such a certificate may be at a disadvantage in terms of attracting patients. Continuing medical

\section{WHAT IS ALREADY KNOWN ON THIS TOPIC}

Hypertension is a major public health problem globally

Doctors in Pakistan have been shown to have serious gaps in knowledge of and approach to treatment of patients with hypertension

Lack of adherence to antihypertensive drugs contributes to poor rates of blood pressure control

\section{WHAT THIS STUDY ADDS}

Special training of general practitioners with a simple educational package on management of hypertension led to significantly improved adherence among communities in Pakistan

Simple interventions emphasising good communication between doctors and patients should be adopted by other developing countries

education courses are lacking in Pakistan, and opportunities for further education seem to be welcome. ${ }^{10}$ This model of training of general practitioners could be usefully adopted in other developing countries as well, especially in Indo-China. Thus, our findings have implications for more than a third of the world's population.

\section{Conclusion}

Our study is the first to show that simple training of general practitioners in management of hypertension has a positive impact on adherence to antihypertensive drugs at a population level in a developing country. This in turn improved blood pressure control. A key determinant was an explanation provided by the general practitioner of the purpose of drugs. Our findings provide strong support for continuing medical education in the management of hypertension, which should include simple yet important messages such as better communication between doctor and patient. Such interventions are ideal for the developing world, where the burden of hypertension is on the increase and resources are scarce.

Other members of the Hypertension Research Group: S Badruddin, A Hameed, F Jafary, A Khan, M Karim, A Gilani, S Hashmi, S Jessani, R Bux, Z Qadri, M Saleem, P Cosgrove, and A Khan (Aga Khan University, Karachi, Pakistan); N Poulter (Imperial College, London)

Contributors: $\mathrm{THJ}, \mathrm{NC}$, and JH were responsible for the study concept and design. All authors obtained funding. NNQ was involved in data acquisition under THJ's supervision. NNQ, JH, and THJ did the statistical analysis. NNQ and THJ drafted the manuscript, and all authors critically revised it for important intellectual content. $\mathrm{TH}$ J is the guarantor.

Funding: Wellcome Trust, UK (THJ, NC, JH) and Aga Khan University Research Council (NNQ). MEMS bottles were provided by Aardex, Switzerland.

Competing interests: None declared.

Ethical approval: Aga Khan University Ethics Review Committee.

1 Lopez AD, Mathers CD, Ezzati M, Jamison DT, Murray CJ. Global and regional burden of disease and risk factors, 2001: systematic analysis of population health data. Lancet 2006;367:1747-57.

2 He J, Gu D, Wu X, Reynolds K, Duan X, Yao C, et al. Major causes of death among men and women in China. N Engl J Med 2005;353:1124-34.

3 Hypertension Detection and Follow-up Program Cooperative Group. Five-year findings of the hypertension detection and follow-up 
program: I. Reduction in mortality of persons with high blood pressure, including mild hypertension. JAMA 1997;277:157-66.

4 Hajjar I, Kotchen TA. Trends in prevalence, awareness, treatment, and control of hypertension in the United States, 1988-2000. JAMA 2003;290:199-206.

5 Wang Z, Wu Y, Zhao L, Li Y, Yang J, Zhou B. Trends in prevalence, awareness, treatment and control of hypertension in the middle-aged population of China, 1992-1998. Hypertens Res 2004; $27: 703-9$.

6 Jafar TH, Levey AS, Jafary FH, White F, Gul A, Rahbar MH, et al. Ethnic subgroup differences in hypertension in Pakistan. $J$ Hypertens 2003;21:905-12.

7 Degli Esposti E, Di Martino M, Sturani A, Russo P, Dradi C, Falcinelli S, et al. Risk factors for uncontrolled hypertension in Italy. J Hum Hypertens 2004;18:207-13.

8 Dusing R. Overcoming barriers to effective blood pressure control in patients with hypertension. Curr Med Res Opin 2006;22:1545-53.

9 Krousel-Wood M, Thomas S, Muntner P, Morisky D. Medication adherence: a key factor in achieving blood pressure control and good clinical outcomes in hypertensive patients. Curr Opin Cardiol 2004; 19:357-62.

10 Jafar TH, Jessani S, Jafary FH, Ishaq M, Orakzai R, Orakzai S, et al. General practitioners' approach to hypertension in urban Pakistan: disturbing trends in practice. Circulation 2005;111:1278-83.

11 Hyman DJ, Pavlik VN, Vallbona C. Physician role in lack of awareness and control of hypertension. J Clin Hypertens (Greenwich) 2000;2:324-330.

12 Sisson SD, Rastegar D, Rice TN, Prokopowicz G, Hughes MT. Physician familiarity with diagnosis and management of hypertension according to JNC 7 guidelines. J Clin Hypertens (Greenwich) 2006;8:344-50.

13 Hagemeister J, Schneider CA, Barabas S, Schadt R, Wassmer G, Mager G, et al. Hypertension guidelines and their limitations-the impact of physicians' compliance as evaluated by guideline awareness. J Hypertens 2001;19:2079-86.

14 Jafar TH, Jafary FH, Jessani S, Chaturvedi N. Heart disease epidemic in Pakistan: women and men at equal risk. Am Heart / 2005;150:221-6.

15 Campbell M, Grimshaw J, Steen N. Sample size calculations for cluster randomised trials. J Health Serv Res Policy 2000;5:12-6.

16 Chobanian AV, Bakris GL, Black HR, Cushman WC, Green LA, Izzo JL Jr, et al. The seventh report of the Joint National Committee on Prevention, Detection, Evaluation, and Treatment of High Blood Pressure: the JNC 7 report. JAMA 2003;289:2560-72.

17 Williams B, Poulter NR, Brown MJ, Davis M, McInnes GT, Potter JF, et al. British Hypertension Society guidelines for hypertension management 2004 (BHS-IV): summary. BMJ 2004;328:634-40.
18 Fahey T, Schroeder K, Ebrahim S. Interventions used to improve control of blood pressure in patients with hypertension. Cochrane Database Syst Rev 2006;(4):CD005182.

19 Aucott JN, Pelecanos E, Dombrowski R, Fuehrer SM, Laich J, Aron DC. Implementation of local guidelines for cost-effective management of hypertension: a trial of the firm system. J Gen Intern Med 1996;11:139-46.

20 Hamilton GA. Measuring adherence in a hypertension clinical trial. Eur J Cardiovasc Nurs 2003;2:219-28.

21 Lee JY, Kusek JW, Greene PG, Bernhard S, Norris K, Smith D, et al. Assessing medication adherence by pill count and electronic monitoring in the African American study of kidney disease and hypertension (AASK) pilot study. Am J Hypertens 1996;9:719-25.

22 Eldridge SM, Ashby D, Feder GS, Rudnicka AR, Ukoumunne OC. Lessons for cluster randomized trials in the twenty-first century: a systematic review of trials in primary care. Clin Trials 2004;1:80-90.

23 Cochran GW, Snedecor W. Statistical methods. 7th ed. Ames, IA: Iowa State University Press, 1980.

24 Beaglehole R. Responding to the dual burden of nutritional diseases. Asia Pac J Clin Nutr 2004;13(suppl):S1.

25 Ahmad K, Jafar TH. Prevalence and determinants of blood pressure screening in Pakistan. J Hypertens 2005;23:1979-84.

26 Liu GG, Zhao Z, Cai R, Yamada T. Equity in health care access to: assessing the urban health insurance reform in China. Soc Sci Med 2002;55:1779-94.

27 Singh P, Yadav RJ, Pandey A. Utilization of indigenous systems of medicine \& homoeopathy in India. Indian J Med Res 2005;122:137-42.

28 Krakoff LR. The ASH specialists program: a progress report. Am J Hypertens 2002;15:577-9.

29 Bansal N, Tendler BE, White WB, Mansoor GA. Blood pressure control in the hypertension clinic. Am J Hypertens 2003;16:878-80.

30 Goeman DP, Hogan CD, Aroni RA, Abramson MJ, Sawyer SM, Stewart K, et al. Barriers to delivering asthma care: a qualitative study of general practitioners. Med J Aust 2005;183:457-60.

31 Mechanic D. How should hamsters run? Some observations about sufficient patient time in primary care. BMJ 2001;323:266-8.

32 Lane DA, Lip GY. Ethnic differences in hypertension and blood pressure control in the UK. OJM 2001;94:391-6.

33 Wolf-Maier K, Cooper RS, Kramer H, Banegas JR, Giampaoli S, Joffres MR, et al. Hypertension treatment and control in five European countries, Canada, and the United States. Hypertension 2004;43:10-7.

Accepted: 20 September 2007 